Rosa Cetro et Lorella Sini (éds), 2021. Fake news, rumeurs, intox... Stratégies et visées discursives de la désinformation (Paris : L'Harmattan)

\title{
Lucie Donckier de Donceel
}

\section{(2) OpenEdition Journals}

Electronic version

URL: https://journals.openedition.org/aad/5925

DOI: $10.4000 /$ aad. 5925

ISSN: 1565-8961

Publisher

Université de Tel-Aviv

Electronic reference

Lucie Donckier de Donceel, "Rosa Cetro et Lorella Sini (éds), 2021. Fake news, rumeurs, intox... Stratégies et visées discursives de la désinformation (Paris : L'Harmattan)", Argumentation et Analyse du Discours [Online], 27 | 2021, Online since 14 October 2021, connection on 16 October 2021. URL: http:// journals.openedition.org/aad/5925 ; DOI: https://doi.org/10.4000/aad.5925

This text was automatically generated on 16 October 2021 .

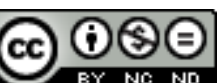

Argumentation \& analyse du discours est mis à disposition selon les termes de la licence Creative Commons Attribution - Pas d'Utilisation Commerciale - Pas de Modification 4.0 International. 


\title{
Rosa Cetro et Lorella Sini (éds), 2021. Fake news, rumeurs, intox... Stratégies et visées discursives de la désinformation (Paris : L'Harmattan)
}

\author{
Lucie Donckier de Donceel
}

\section{REFERENCES}

Rosa Cetro et Lorella Sini (éds), 2021. Fake news, rumeurs, intox... Stratégies et visées discursives de la désinformation (Paris : L'Harmattan), ISBN : 978-2-343-20884-8, 326 pages

1 L'ouvrage de Rosa Cetro et Lorella Sini s'ouvre sur une présentation du volume par les deux auteures. Après avoir rappelé le cadre dans lequel ce projet a vu le jour ${ }^{1}$, elles s'attèlent à une double tâche : délimiter une définition générique et une traduction française pour le syntagme anglais fake news et, plus globalement, retracer les différents contextes dans lequel cette notion a évolué et évolue encore ${ }^{2}$.

De fait, les deux chercheuses soulignent l'émergence de ce terme anglophone dans une période marquée par les phénomènes de post-vérité et de défiance généralisée, bien qu'elles insistent aussi sur le fait que la propagation de fausses nouvelles, rumeurs et opérations de désinformation ne sont pas, en soi, des pratiques nouvelles. En effet, ce qui bouleverse l'équilibre de l'écosystème de l'information ces dernières années, ce sont principalement la vitesse de diffusion des informations et la démultiplication des orateurs et auditoires rendues possible par les nouvelles technologies, plateformes et multimédias (Cetro et Sini, Présentation, 11-17). Cette constatation sera d'ailleurs reprise par la plupart des auteur(e)s impliqué(e)s dans ce volume.

Plus précisément, l'ouvrage est construit en trois volets. Les contributions de la première partie se centrent sur la question de la manipulation de l'information à 
travers l'analyse de discours et de contre-discours. La question complexe de la "vérité » apparaît également en filigrane dans cette partie.

Les contributions de la seconde partie offrent quant à elles une réflexion sur le rapport de continuité entre propagande et intox à travers le temps et dans deux domaines spécifiques : la politique et la religion.

Enfin, les contributions de la troisième partie, plus brèves, se concentrent sur le rapport entre intox et discours conspirationnistes. Prises dans leur globalité, chacune des contributions présentes dans cet ouvrage soulignent et illustrent, à leur façon, la pertinence des études en sciences du langage pour tenter non seulement, de comprendre le monde qui nous entoure, mais aussi, proposer des pistes de réflexions pour aller à l'encontre des phénomènes qui portent atteinte aux fondements du vivreensemble que nous expérimentons de nos jours.

6 La partie "Enjeux sociaux, discours et contre-discours " ${ }^{3}$ se révèle essentielle pour poser le cadre méthodologique et théorique propre aux sciences du langage et pour appréhender, dans cette optique, la notion d'intox. Le premier auteur, Julien Longhi, en s'inscrivant dans le sillage de G.-E. Sarfati, propose de concevoir les intox comme des objets discursifs (Sarfati 2008). Plus généralement, il plaide en faveur d'une revalorisation de l'analyse discursive comme cadre épistémologique pour, non seulement, pouvoir cartographier l'information mais aussi pour pouvoir appréhender le monde qui nous entoure ; ce postulat est illustré dans la suite.

7 Les deux contributions suivantes soutiennent toutes deux ce propos en soulevant, à travers des cas d'études précises - la première aborde la question des Portier papers et la seconde se concentre, elle, sur la communication autour d'un projet d'exploitation de gaz de charbon en Lorraine - des questions qui concernent le fondement même de nos démocraties et les attitudes adoptées par les différents acteurs (citoyens, médias, politiques, experts scientifiques, etc.) au sein de celles-ci. Dans cette perspective, François Allard-Huver interroge le rôle du chercheur dans nos sociétés et Marieke Stein souligne la nécessité d'une information vraie pour permettre le débat et ne pas déposséder le citoyen de sa capacité à se forger une opinion et l'exprimer. Ces considérations, qui permettent de distinguer la controverse scientifique de la polémique, font largement écho à notre actualité et aux dernières réflexions du physicien Etienne Klein (Klein 2020a, 2020b). Elles nous aident à comprendre en quoi le passage de l'un à l'autre est une question éminemment politique.

8 Ces premières contributions sont enrichies par la réflexion d'Alessandra Rollo qui met, quant à elle, l'accent sur l'impact des intox sur un secteur particulier et moins fréquemment mobilisé quand on aborde cette problématique : le secteur économique. Plus spécifiquement, à l'instar des deux contributions précédemment citées, elle explique en quoi les intox peuvent être mobilisées dans une véritable entreprise de sabotage à l'encontre, ici, d'organismes financiers.

9 Les trois autres contributions présentes dans cette partie sont conçues dans un rapport de complémentarité avec les quatre premières. En effet, elles peuvent être abordées comme des réflexions autour de possibles moyens techniques pour lutter contre la propagation des intox. Les trois auteur(e)s proposent, chacun(e) à leur manière de lutter contre la propagation des intox en reconsidérant positivement le rôle de certains experts de la vie publique. D'une part, Dominique Dias et Silvia Modena soutiennent qu'une possible solution face à la prolifération des intox se situe dans une revalorisation du métier de journaliste. Le premier auteur voit dans la pratique du fact- 
checking une redécouverte des principes fondamentaux de cette profession. Et à son instar, la seconde auteure soutient que la ressource pédagogique constituée par la bande dessinée Fred se méfie des fausses infos ${ }^{4}$ participe d'un même effet: rendre ses lettres de noblesse au journalisme et aux pratiques qui sous-tendent ce métier. D'autre part, Valérie Delavigne propose quant à elle de revaloriser la figure d'expert en déconstruisant l'apriori plutôt négatif véhiculé à l'encontre des forums. En effet, selon l'auteure, les forums sont le lieu de (re)construction et d'expression d'une forme de confiance envers deux figures d'experts particulières, qu'elle nomme l'expert technique, typiquement, le praticien reconnu par ses pairs et dans la vie publique et l'expert " de terrain », celui qui a expérimenté de première main et offre un soutien qui relève plutôt du réconfort. La distinction et définition de ces deux figures d'expertise est esquissée à partir de l'analyse de forums de discussion de patients autour de la question du cancer.

10 La seconde partie de l'ouvrage est constituée de différentes études de cas qui, bien qu'elles se distinguent d'un point de vue chronologique et géographique, s'ancrent toutes dans les deux mêmes domaines : le champ du politique et du religieux. En allant de la rumeur à l'intox, ces six contributions illustrent donc clairement les enjeux politiques et sociétaux soulignés auparavant. Au-delà, elles préparent aussi le terrain pour la troisième partie de l'ouvrage qui traite des rapports entre intox et théories conspirationnistes.

11 Les deux premières contributions issues de cette partie sont celles de Nolwenn LorenziBailly et Claudine Moïse et d'Alida Maria Silletti. En effet, les auteures de la première et celle de la seconde présentent un rapport paradoxal aux informations, elles soulignent toutes les trois ne pas exposer des cas d'intox mais plutôt de "malinformation ", un concept faisant référence à la manipulation, à visée de propagande, d'informations qui sont vraies. Les trois chercheuses soulignent comment, à partir de vérités qui sont présentées comme objectives et rationnelles, respectivement, la parole des experts occidentaux - nous soulignons ${ }^{5}$ - et des données chiffrées ${ }^{6}$, une opération de manipulation peut s'orchestrer. Dans les deux cas, ces vérités sont mises au service d'un discours de propagande idéologique. Mais, et c'est important, bien que présentées comme neutres, elles sont ensuite manipulées pour servir les intentions, soit de l'État Islamique (EI), soit du Front National, devenu Rassemblement National en France (FN/ RN). Dans les deux cas, les pratiques manipulatoires sont les suivantes : on floute la source, on sort les affirmations/chiffres de leur contexte. On note cependant une différence entre les deux cas de «malinformation » ici étudiés. La propagande de l'EI illustre un rapport paradoxal à l'argument d'autorité que peut représenter la parole d'un expert: la parole des experts occidentaux est d'une part valorisée, présentée comme objective et rationnelle - de façon très terre-à-terre : elle sert à légitimer l'existence même de l'EI. Et d'autre part, ces mêmes experts sont en permanence dévalorisés car considérés comme des "mécréants". Nous sommes donc dans un rapport de manipulation ambigu qui se rapproche de certains procédés rhétoriques que l'on repère dans les discours conspirationnistes : la contradiction n'est pas un frein à la persuasion (Danblon et Nicolas 2010). Au contraire, l'usage des chiffres donné par le $\mathrm{FN} / \mathrm{RN}$ dans leurs tracts à propos de l'immigration relèverait plutôt d'une manipulation classique des données : on accumule et juxtapose des informations pour créer des liens de causalités entre ces données qui, a priori, n'ont pas de réel rapport entre elles. Dans des cadres certes différents, ces deux contributions mettent l'accent sur la manipulation de l'information dans un contexte de propagande idéologique. Il nous 
semble donc intéressant de proposer, en vis-à-vis, la lecture de la contribution d'Alice Hélène Burrows dans ce volume.

En effet, dans sa contribution, l'auteure retrace l'évolution d'une propagande politique assumée, voire considérée comme un outil pédagogique, vers une propagande considérée comme une pratique négative, voire source de discours complotiste. Cette analyse est réalisée à partir des Bulletins de Guerre produits par l'Alliance française durant le premier conflit mondial et se présente comme un travail issu d'une réflexion interdisciplinaire : l'auteure mobilise les outils de la micro-histoire (Ginzburg 1989) et de l'histoire connectée (Douki et Minard 2007) et ceux de l'analyse discursive, qu'elle décline sur deux plans distincts mais complémentaires : le champ de la lexicographie et celui de la rhétorique.

L'étude de l'évolution d'un terme est également le propos de la contribution de Kamila Oulebsir-Oukil. L'auteure retrace comment le terme "rumeur" à propos d'un événement précis et dans un cadre particulier évolue et donne naissance à différentes réactions publiques et politiques. L'analyse discursive qu'elle propose permet de comprendre finement en quoi la qualification du syntagme étudié et le vocable qui l'entoure soulignent les différents statuts de ce même événement ${ }^{7}$.

Pour clôturer la deuxième partie de l'ouvrage, nous proposons de regrouper en un même commentaire les contributions de Laura Calabrese, Jeoffrey Gaspard et Pascal Froissart ainsi que de Mélanie Gantier. En effet, ces deux contributions se penchent sur une figure désormais incontournable lorsqu'on aborde la question des fake news, nouveaux médias et faits alternatifs : Donald Trump. Tandis que la contribution à six mains aborde la question de l'usage de Twitter par l'ex-président américain, la dernière contribution aborde, quant à elle, le rapport, que l'on pourrait presque qualifier de personnel, de Trump aux médias. Les premier(e)s auteur(e)s soulignent en quoi, à travers l'étude d'un corpus de Tweets récolté entre 2014 et 2018, l'usage de l'expression fake news reflète la construction d'un discours maîtrisé sur les médias. En effet, que ce soit d'abord en tant qu'homme d'affaire, ensuite surtout, en tant que candidat à la Présidence et enfin comme Président, les auteur(e)s soulignent que le terme fake news est utilisé principalement de trois façons par Donald Trump : pour décrédibiliser les médias établis, pour dévaloriser ses adversaires et pour contrecarrer les accusations, qu'il qualifie d'opérations de désinformation, dont il fait l'objet. À travers ce parcours lexicographique, les trois auteur(e)s, arrivent à l'intéressante et inhabituelle conclusion selon laquelle le Président sortant aurait fait preuve d'un usage réfléchi et construit des réseaux sociaux, Twitter en premier lieu, durant sa campagne et son mandat. Cette hypothèse est, nous le disions, à contre-courant de la tendance générale qui consiste à qualifier les sorties médiatiques de l'ancien Président d'irrationnelles et d'irréfléchies. C'est également cette proposition que nous retrouvons en filigrane dans la réflexion de Mélanie Gantier. En effet, l'auteure soutient que l'usage du terme fake news par l'ancien Président lui permet non seulement de construire un discours émotionnellement négatif, mais illustre surtout une grande maitrise de l'information qu'il diffuse. Dans cette contribution, la textométrie est mobilisée et mise en parallèle avec une réflexion sur les émotions. Ces deux outils permettent à l'auteure, à travers l'étude du vocable et des métaphores qui entourent l'expression fake news, de délimiter le rapport que l'ex-Président entretient avec cette étiquette mais aussi avec les médias de façon plus générale. Bien que Mélanie Gantier, à l'instar de Calabrese, Gaspard et Froissart, soutienne que le terme de fake news est utilisé par Trump pour 
qualifier les médias qui lui sont préjudiciables, et donc contrecarrer les prétendues accusations de "mésinformation » à son égard et pour attaquer ses adversaires, elle propose une conclusion différente de celle des trois auteur(e)s précédent(e)s. Selon elle, le rapport particulier que l'ex-Président entretient avec les médias et certaines expressions qui circulent reflète l'idée selon laquelle, pour Trump, le terme de fake news serait pratiquement équivalent à " une attaque malintentionnée envers sa personne ». La troisième partie, «Fake news et théories du complot ", plus brève, est constituée de trois contributions, rédigées par Rosa Cetro, Francesco Attruia et Lorella Sini et Clémentine Hougue.

Cette troisième partie se démarque légèrement des thématiques précédemment abordées et renvoyant au titre de l'ouvrage: les intox, rumeurs et fake news. Cette dernière section aborde en effet la question des théories du complot. Globalement, la première contribution, à l'instar de l'introduction des deux éditrices et de la réflexion de Julien Longhi, pose le cadre. À travers l'étude des définitions qu'en proposent les dictionnaires, Rosa Cetro définit clairement ce que signifient les termes « complot » et « conspiration » et l'expression « théorie du complot» (TC). Ensuite, le cadre défini, les deux contributions suivantes explorent chacune un aspect distinct des TC : la première, la mobilisation et l'apparition des TC sur la scène politique et l'autre, une proposition quant à l'explosion des TC sur la scène publique ces dernières années. Bien évidemment, ces contributions font écho à l'évolution des termes étudiés par Rosa Cetro et toutes les trois sont en lien avec le concept d'intox, présenté soit comme nourrissant les discours complotistes, soit au contraire, forgé sur la base de ces derniers.

Dans la première contribution, le parcours lexicographique et dictionnairique (Pruvost 2010) proposé par Cetro permet, d'une part, de faire apparaitre qu'une définition d'un(e) mot/notion est toujours en lien avec le contexte historique et sociétal dans lequel elle se développe et, d'autre part, de faire le distinguo entre des notions qui, bien qu'elles fonctionnent dans des rapports de réciprocité, ne sont néanmoins pas automatiquement des synonymes. Pour retracer l'évolution des termes étudiés, l'auteure se propose de parcourir les dictionnaires de langue française sur trois grandes périodes : l'Ancien Régime, le $19^{\mathrm{e}}$ siècle et les $20^{\mathrm{e}}$ et $21^{\mathrm{e}}$ siècles. Voici ce qu'il ressort de ce parcours : lors de l'Ancien Régime, le mot «complot» se rattachait plutôt à une action entreprise à l'encontre d'une personne, d'un individu, tandis que celui de " conspiration » désignait une action visant le pouvoir. Ensuite, au $19^{\mathrm{e}}$ siècle, les deux termes deviennent synonymes et se voient attribuer un statut juridique. Enfin, le $20^{\mathrm{e}}$ siècle est marqué par un dédoublement de la définition du terme complot/ conspiration : il désigne à la fois un caractère juridique et technique du terme et une acceptation plus générique. Globalement, l'auteure souligne donc un basculement d'une dimension privée et individuelle du complot à une dimension, que nous connaissons actuellement, davantage collective et globale. C'est dans le cadre de cette dimension collective que l'expression "théorie du complot» et son dérivé «complotiste» voient le jour. Ces deux termes, désormais largement diffusés font référence, comme le propose Le Petit Robert de 2019, à l'idée « selon laquelle l'opinion est manipulée par un groupe de conspirateurs pour cacher les circonstances réelles de certains événements » et l'adjectif dérivé "complotiste », quant à lui, "désigne un défenseur d'une théorie du complot $»$. 

«Grand Remplacement» pour le corpus francophone et sostituzione etnica pour le corpus italien. Ces deux formulent se manifestent dans des TC à dimension politique forte et partagent de nombreux points communs : toutes les deux reflètent l'idée d'un remplacement de la société occidentale par les populations issues de culture musulmane ${ }^{8}$, toutes les deux sont portées sur la scène politique par des partis d'extrême droite, toutes deux font le lit de nombreuses intox à caractère raciste et participant d'un climat d'insécurité généralisée, et toutes les deux voient d'abord leur usage et évocation dans les médias avant leur reprise par les politiques. Les auteur(e)s soulignent cependant une différence entre les usages français et italien des expressions consacrées : tandis qu'en France, les politiques y font majoritairement allusion de façon détournée, en Italie, les politiques l'assument explicitement. Une fois les similarités et différences établies entre les deux formules, les auteur(e)s proposent deux conclusions - la première et la principale : ces TC à dimension politique et polémique fortes sont un terrain fertile pour les intox (d'ailleurs, à ce propos, il est intéressant de mettre en parallèle le fonctionnement des intox identifié dans cette contribution et dans celle d'Alida Maria Silleti car certains mécanismes communs sont identifiés). Et la seconde : une hypothèse d'articulation entre le néologisme «intox » et le phénomène des TC. Les intox qualifieraient des faits présumés et les TC seraient la mise en scène de ces faits supposés dans un récit articulé. Cette hypothèse dialogue parfaitement avec les deux autres contributions de cette partie : la contribution précédente démontre que les complots, et donc les discours à leur propos, n'ont pas toujours qualifié des faits présumés: de nombreux complots avérés parsèment l'histoire. La contribution suivante interroge, quant à elle, la question du récit: comment certains récits fictionnels de la fin du $20^{\mathrm{e}}$ siècle ont contribué à la création de récits complotistes articulés.

La contribution qui clôt le volume, rédigée par Clémentine Hougue, interroge la thèse selon laquelle fictions et discours complotistes développent un même modèle narratif, un modèle dessinant les contours de grands récits explicatifs, c'est-à-dire de nouvelles mythologies. Plus précisément, l'auteure soutient que la fiction cinématographique américaine ${ }^{9}$ de la seconde moitié du $20^{\text {e }}$ siècle est un terrain fertile et une source d'inspiration pour l'émergence des TC. L'auteure base son propos sur trois principes méthodologiques précis : la définition d'une TC par Keeley (1999), les quatre principes clefs pour la construction d'un récit complotiste selon Taguieff (2006) et les apports et précisions apportés à ce propos par Bronner (2013) et Danblon et Nicolas (2010). L'auteure insiste cependant bien: les fictions américaines étudiées ne suffisent pas à elles seules pour expliquer l'explosion des TC de ces dernières années. Elle évoque également un sentiment de perte de sens partagé et un manque de confiance envers les experts et les autorités, terreau fertile pour les complotistes. Nous notons cependant que l'évolution très récente des discours conspirationnistes marque peut-être une première double rupture avec les schémas narratifs présents dans les fictions étudiées. D'abord, les complotistes, rattachés auparavant à la figure du héros marginalisé, fonctionnent de plus en plus en communautés structurées ${ }^{10}$. Ensuite, l'idée qu'il serait impossible de lutter contre les forces malveillantes et omnipotentes à l'œuvre nous semble pouvoir être remise en question par l'assaut du Capitole en janvier 2021 par les partisans du mouvement QAnon. 

news, rumeurs, intox et, à moindre échelle, de complot et théorie du complot. Cependant, la volonté de définir ces notions désormais omniprésentes dans notre quotidien est telle que chaque auteur(e) prend le temps de le faire, ce qui provoque parfois un inévitable effet de répétition. Cela étant dit, cet ouvrage est surtout un beau plaidoyer pour l'analyse du discours et l'enjeu que cette discipline représente pour comprendre les phénomènes discursifs contemporains. Nous pouvons ainsi conclure avec Julien Longhi : « Il y a donc un enjeu fondamental à sensibiliser les citoyens à l'analyse du discours, ainsi qu'à la production de discours critiques, et à inviter les spécialistes de l'analyse du discours à investir les espaces d'information et de débat » (Longhi $2021: 38$ ).

\section{BIBLIOGRAPHY}

Charaudeau, Patrick. 2009. «Il n'y a pas de société sans discours propagandiste », Ollivier Yaniv, C. \& M. Rinn (éds), Communication de l'État et gouvernement social (Grenoble : P. U. de Grenoble)

Danblon, Emmanuelle \& Lois NICOLAS (éds). 2010. Les rhétoriques de la conspiration (Paris : CNRS Editions)

Douki, Claire \& Pierre Minard. 2007). « Histoire globale, histoires connectées : un changement d'échelle historiographique ? ", Revue d'Histoire moderne et contemporaine 54-4bis, 7-21

Ginzburg, Carlo. 1989. Mythes, emblèmes et traces. Morphologie et histoire (Paris : Flammarion)

Klein, Etienne. 2020a. « Je ne suis pas médecin, mais je ... », Tracts de crise 25 (Paris : Gallimard.)

Klein, Etienne. 2020b. « Le goût du vrai », Tracts 17 (Paris : Gallimard)

Pruvost, Jean. 2010. « La traque lexicographique et dictionnairique : du loup au chat en passant par le vin, le mariage et le citoyen », Ela. Études de Linguistique appliquée 157, 103-110

Sarfati, Georges-Elia. 2008. «Pragmatique linguistique et normativité : remarques sur les modalités discursives du sens commun », Langages 170, 92-108

Taguieff, Pierre-André. 2016. Pensée conspirationniste et « théories du complot » (Toulouse : UPPR)

\section{NOTES}

1. Ce volume recueille une sélection des contributions présentées lors de deux journées d'études tenues à l'Université de Pise en octobre 2018. Elles portaient sur l'analyse du phénomène de la désinformation au prisme des sciences du langage et concordaient, par un heureux hasard avec la recommandation publiée par la DGLFLF à propos de la traduction française du syntagme fake news. Cet ouvrage est également enrichi de contributions inédites.

2. Nous utiliserons dans ce compte-rendu le néologisme intox pour traduire le terme de fake news.

3. Nous indiquons au lecteur que, bien que nous respections l'organisation du volume en trois parties, la présentation que nous faisons des différentes contributions au sein de chacune de ces 
parties ne suit pas automatiquement l'ordre de l'ouvrage. Nous nous référons aux différentes contributions grâce aux noms des auteur(e)s.

4. Bande dessinée « Fred se méfie des fausses infos. Faites comme Fred. Suivez ces 7 conseils pour apprendre à vérifier l'information! », originellement rédigée en portugais a ensuite été traduite en différentes langues. La version française a été publiée en ligne, en avril 2018 par "Les décodeurs du Monde", une équipe du journal Le Monde. Disponible en ligne : https:// www.lemonde.fr/les-decodeurs/article/2018/04/05/fred-se-mefie-des-fausses-infos-une-bdpour-apprendre-a-verifier-l-information_5281240_4355770.html (consulté le 23 juillet 2021)

5. La contribution de N. Lorenzi-Bailly et C. Moïse porte sur l'analyse d'un corpus bien particulier : l'étude discursive de la rubrique «Dans les mots de l'ennemi » de la revue francophone Dar-AlIslam, outil de propagande de l'état Islamique. Cette étude porte sur dix numéros parus entre décembre 2014 et août 2016.

6. La contribution d'Alida Maria Silletti porte sur les données chiffrées reprises dans les tracts du FN/RN sur l'immigration entre 2008 et 2019.

7. L'événement étudié ici est, en Algérie, l'annonce faite fin 2017 d'une possible cinquième candidature de la part du président sortant Abdelaziz Bouteflika. Cette rumeur sera confirmée dans les mois suivants.

8. Renaud Camus publie en France, en 2011, Le Grand Remplacement qui évoque l'idée d'un remplacement de la population française, et par extension occidentale, par des populations issues de culture musulmane. En Italie, cette même idée est au départ diffusée par les écrits d'Oriana Fallaci qui, elle, se base sur ce que l'on nomme le «plan Kalergi ». Ce «plan» fait allusion au nom et aux écrits d'un philosophe autrichien à l'origine d'un soi-disant plan d'immigration massive d'origine africaine et asiatique en Europe.

9. L'auteure fait référence, entre autres, à Matrix des frères Wachowski (1999), JFK d'Oliver Stone (1991) ou encore au Da Vinci code de Dan Brown (2003).

10. À ce propos, voir à titre d'exemple le réseau en ligne "Solidarita " mis en place par le conspirationniste Jean-Jacques Crèvecoeur qui a pour objectif de permettre aux différents partisans d'une même théorie du complot de se retrouver physiquement et de s'organiser pour la «lutte », par zone de résidence.

https://solidarita.net/ ainsi queles vidéos produites par Crèvecoeur à propos de son réseau sur son canal fulllifechannel : https://fulllifechannel.com/channel/Solidarita (consulté le 26 juillet 2021)

\section{AUTHORS}

\section{LUCIE DONCKIER DE DONCEEL}

Università degli Studi di Palermo et Université libre de Bruxelles 